\title{
Riskometric assessment of factors affecting population health in situational analysis features of cytochemical indicators of activity circulating and tissue leukocytes and oxidative stress as a factor of chronic inflammation
}

Sokolovska Irina $^{\mathrm{a}}$, Kutsak Alla ${ }^{\mathrm{a}}$, Gordienko Lyudmila ${ }^{\mathrm{b}}$, Bulanov Valerii ${ }^{\mathrm{c}}$, Hryshyna Tetiana $^{\mathrm{c}}$, Zarytska Valentyna ${ }^{\mathrm{c}}$, Plakhotnik Olga ${ }^{\mathrm{d}}$, Semeniv Igor $^{\mathrm{e}}$, Kotuza Andriy ${ }^{\mathrm{e}}$, Zazirnyi Igor $^{\mathrm{e}}$, Kmetyuk Yaroslav $^{\mathrm{e}}$, Kovtun Roman ${ }^{\mathrm{f}}$

Department of Hygiene, Zaporizhzhya State Medical University, avenue Mayakovsky 26, Zaporizhzhya 69035, Ukraine Zaporizhzhya State Medical University ${ }^{a}$, Clinical Hospital "Feofania" the State Management of Affairs, Kyiv ${ }^{\mathrm{b}}$,e , Classic Private University ${ }^{\mathrm{c}, \mathrm{f}}$, Ukraine Kyiv National University named after Taras Shevchenko ${ }^{d}$

\section{irinasokol10@meta.ua}

Keywords: oxidative stress, leukocytes, chronic inflammation, lipid peroxidation and proteins, mechanisms of phagocytosis.

The study of the mechanism of oxidative stress and regulation of oxygen-dependent processes is important, as the establishment of a complex interaction between oxidative stress, oxidative protein damage and the body's antioxidant system makes it possible to clarify metabolic pathways of disease pathogenesis. In our study, we concluded that in patients with chronic non-specific_inflammatory diseases of the genitals of men and women, the phagocytic activity of polymorphonuclear leukocytes is reduced mainly by the oxygen-dependent mechanism, and tissue - by the oxygen-independent. As an exception in patients with chlamydial infection, the phagocytic activity of tissue polymorphonuclear leukocytes was also reduced by an oxygen-dependent mechanism.

\section{Introduction}

The urgency of the problem of inflammatory diseases of the genital organs, especially caused by sexually transmitted infections, is growing every year. These diseases are one of the most common in the general structure of infectious pathology and pose a serious medical, social and demographic danger. At present, the examination of patients with chronic non-specific inflammatory diseases of the genitals shows a chronic or latent long course, the development of severe complications, which requires a new strategy and tactics from the 
doctor, a new look at the diagnosis and treatment of these diseases (Figure 1).

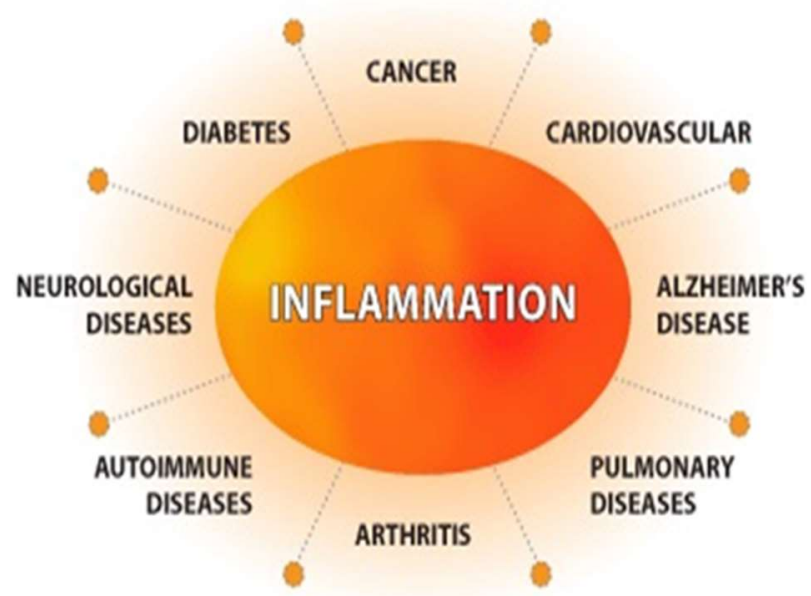

Figure 1. Chronic inflammation and its effects on the entire body.

Immunoinflammatory processes cause lipid imbalance, which can be mostly caused by imbalance of pro- and antioxidant systems or develop by other mechanisms, which plays a major pathogenetic role in the development of endothelial dysfunction and a number of diseases and pathological conditions. Antioxidant system is a set of enzymatic and non-enzymatic factors that protect the cell from free radicals formed.

(Figure

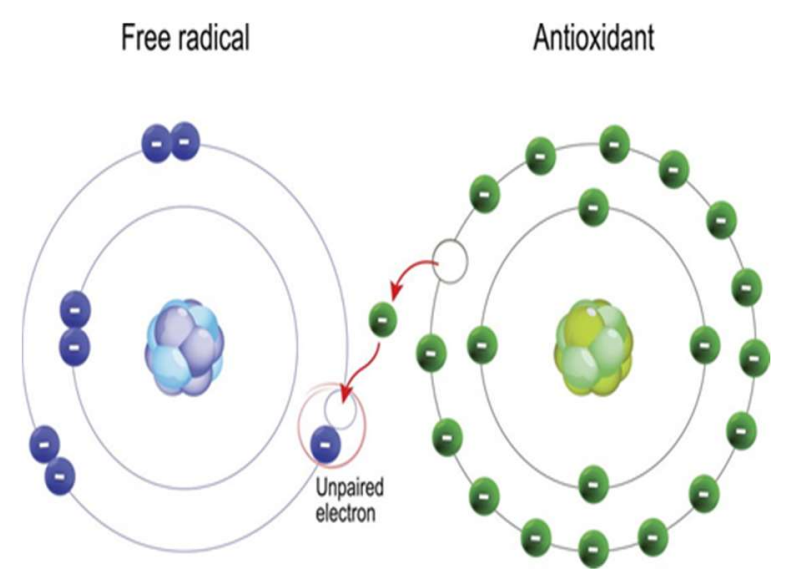

2).

Figure 2. Antioxidant defense mechanism.

Antioxidant enzymes include superoxide dismutase, which catalyses the dismutation of oxygen to hydrogen peroxide, glutathione reductase, glutathione peroxidase, ceruloplasmin, and catalase, which decomposes hydrogen peroxide to water and oxygen molecules. A high degree of association between adverse environmental risk factors (smoking, physical inactivity, malnutrition, overweight) and impaired blood lipids has also been studied and proven. Chronic endoplasmic reticulum stress, oxidative stress, or inflammation have been found to be associated with the initiation and progression of a variety of human diseases in the modern world [1] (Figure 3).

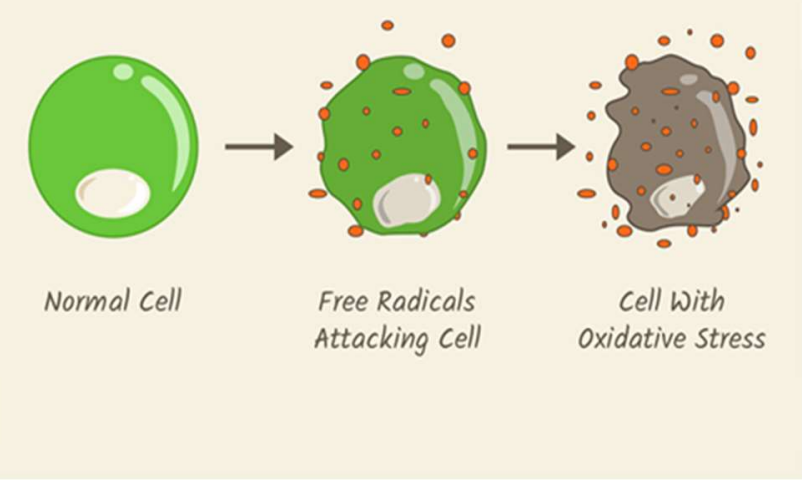

Figure 3. Cell with Oxidative Stress.

The main reason for the failure of the mechanisms of specific and non-specific defense of the body in many diseases and pathological conditions is an imbalance in the system of 


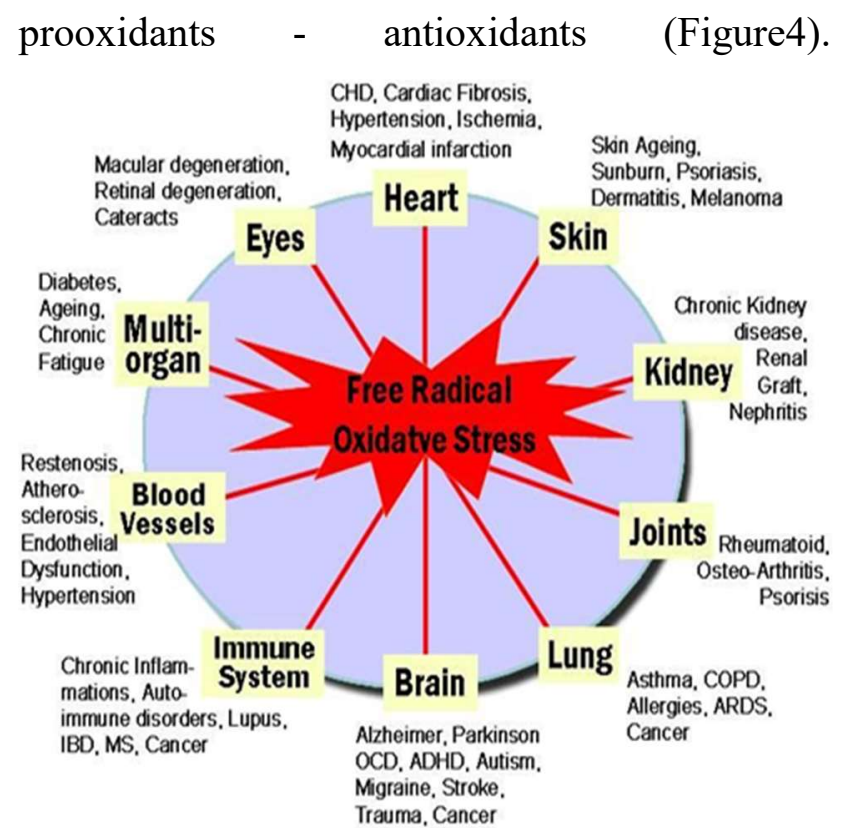

Figure 4. Consequences of the influence of free radicals on organs and systems.

Today, many people face factors that directly or indirectly lead to the formation of excessive amounts of prooxidants (Figure 5).

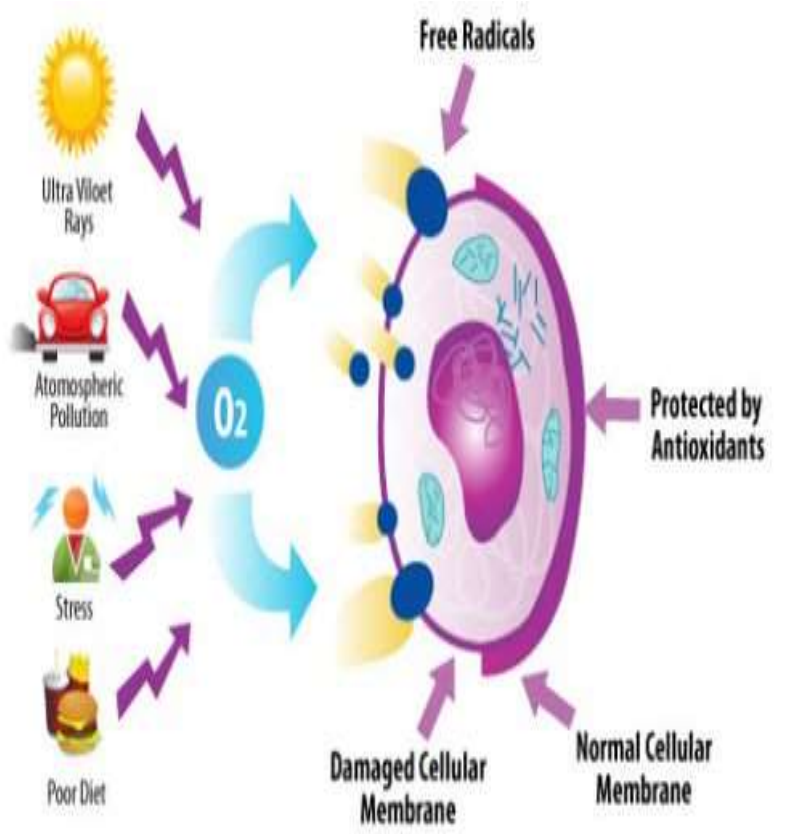

Figure 5. The factors that can lead to the excessive formation of free radicals.

A significant imbalance in it is accompanied by the development of oxidative stress with its pathological components (Figure6).

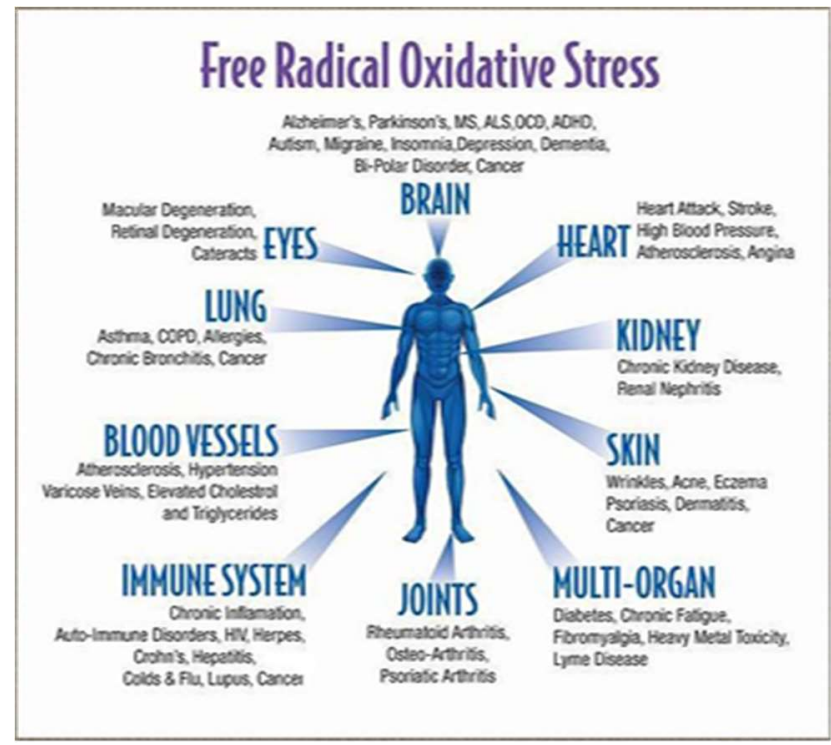

Figure 6. Free Radical Oxidative Stress

Non-enzymatic dismutation of oxygen under certain conditions can produce singlet oxygen, which, not being a free radical, has a high activity. Oxygen can also react with hydrogen peroxide to form a highly active hydroxyl radical. Due to the appearance of free radicals, free radical oxidation of lipids is initiated, which is replaced by reactions with the formation of peroxide radicals and lipid hydroperoxides. Branching reactions lead to the formation of aldehydes, epoxides, ketones, or to interactions with antioxidants.

The presence of oxidative stress has been observed in a number of physiological and many pathological conditions (Figure 7). 


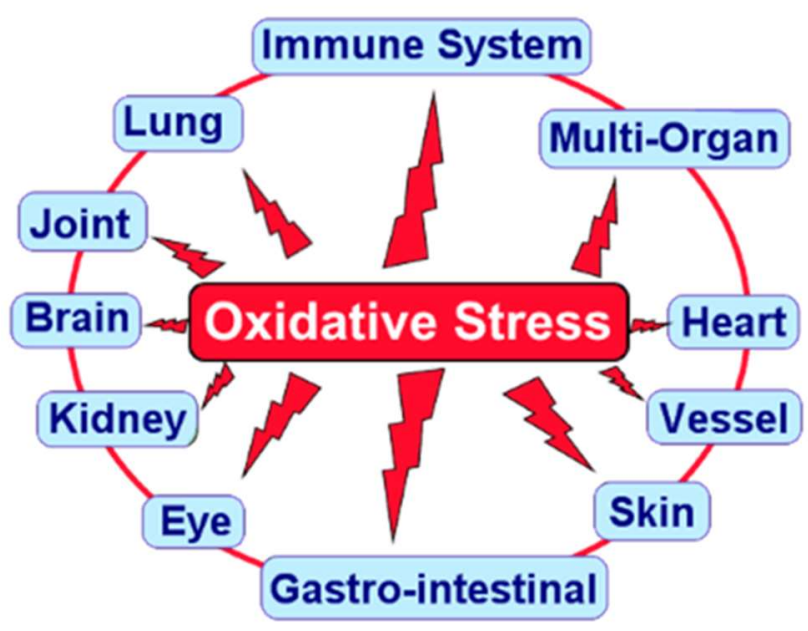

Figure 7. Oxidative stress acts on target organs.

Oxidative stress has been found to be associated with endothelial dysfunction, inflammation, hypertrophy, apoptosis, cell migration, fibrosis, and angiogenesis in relation to vascular remodeling of hypertension [2] (Figure 8).

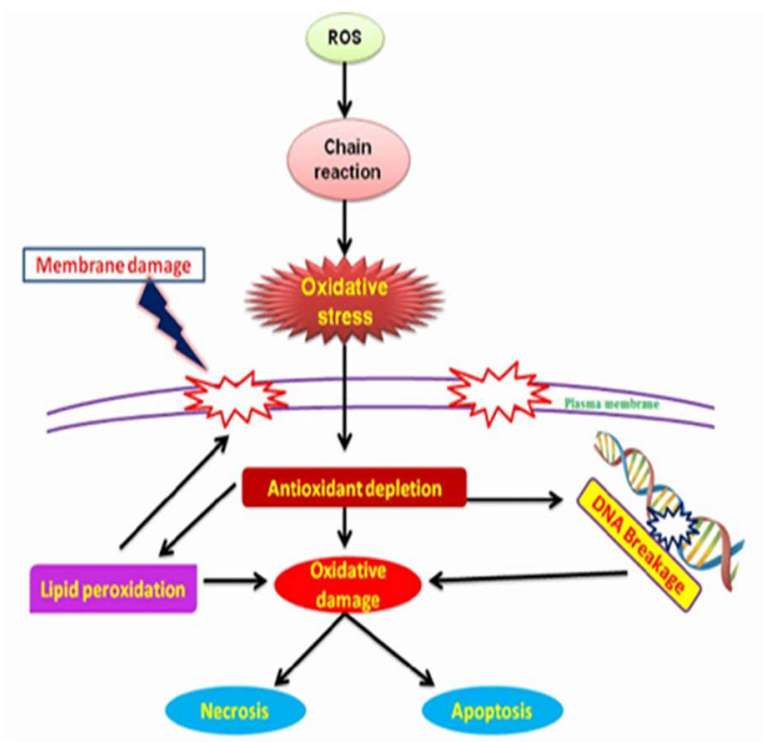

Figure 8. Oxidative damage that leads to cell death.

Oxidative stress induced by generation of excess reactive oxygen species has emerged as a critical, final common mechanism in atherosclerosis [3] (Figure 9).

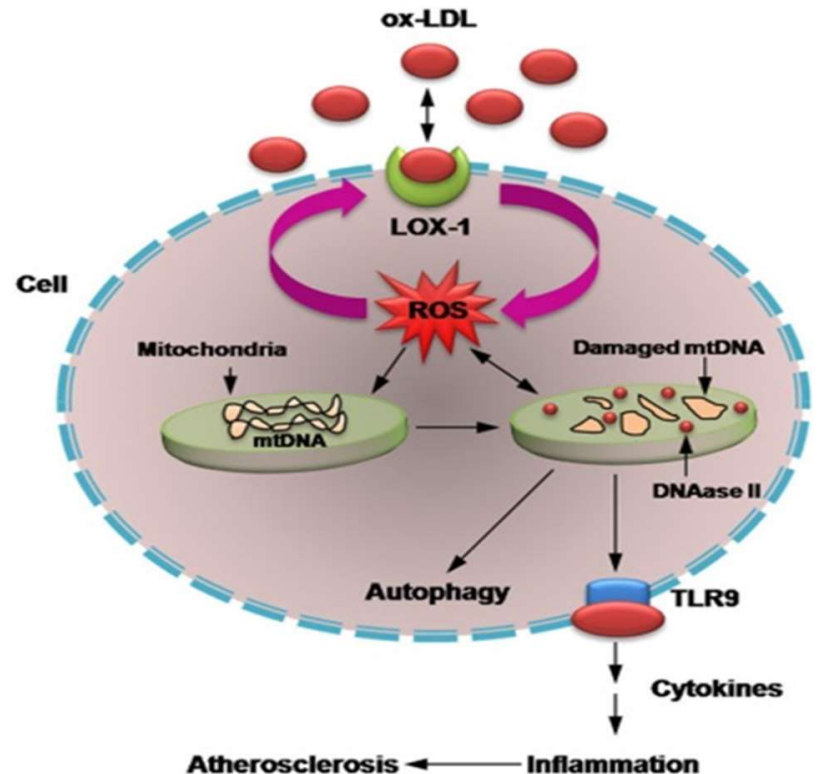

Figure 9. The development of atherosclerosis as a consequence of the effect of free radicals on the cell.

Oxidative stress is defined as an excessive production of reactive oxygenated species that cannot be counteracted by the action of antioxidants, but also as a perturbation of cell redox balance (Figure 10).

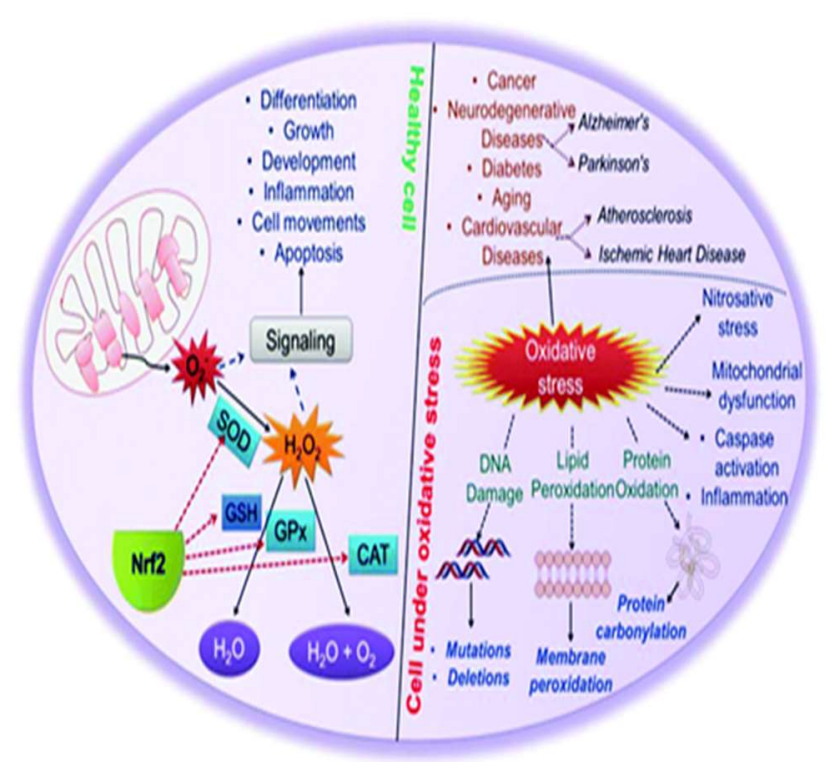

Figure 10. Oxidative stress leads to disruption of physiological processes in the cell.

Reactive oxygenated / nitrogenated species are represented by superoxide anion radical, 
hydroxyl, alkoxyl and lipid peroxyl radicals, nitric oxide and peroxynitrite. [4] Oxidative stress determines structure modifications and function modulation in nucleic acids, lipids and proteins [5] (Figure 11).

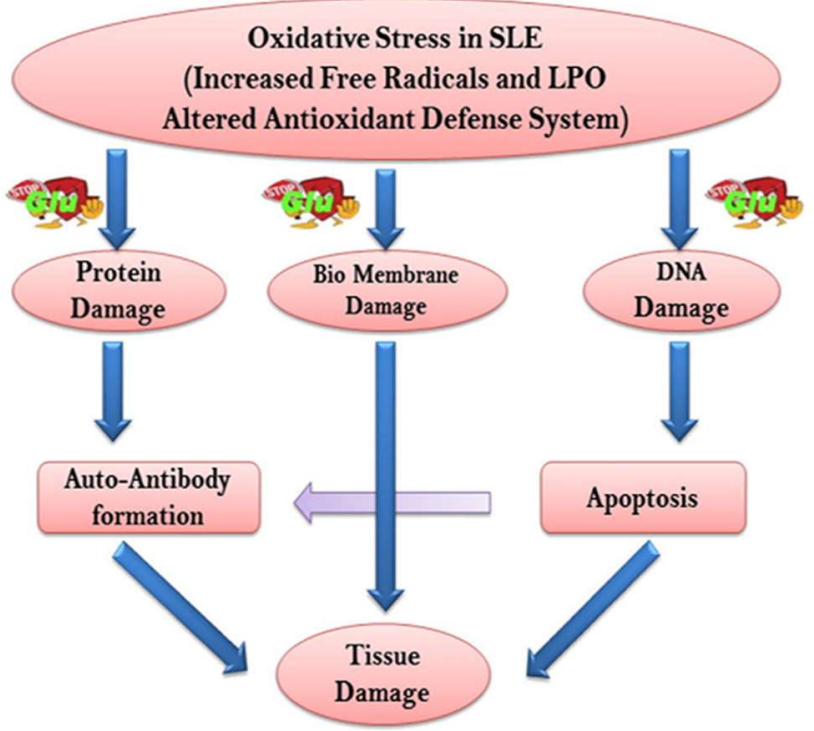

Figure 11. The interaction of oxidative stress and apoptosis in SLE.

Oxidative stress occurs as a result of imbalance between redox processes, under the influence of external or internal factors and leads to oxidative modification of biomolecules, in particular, lipids, proteins and DNA [6] (Figure 12).

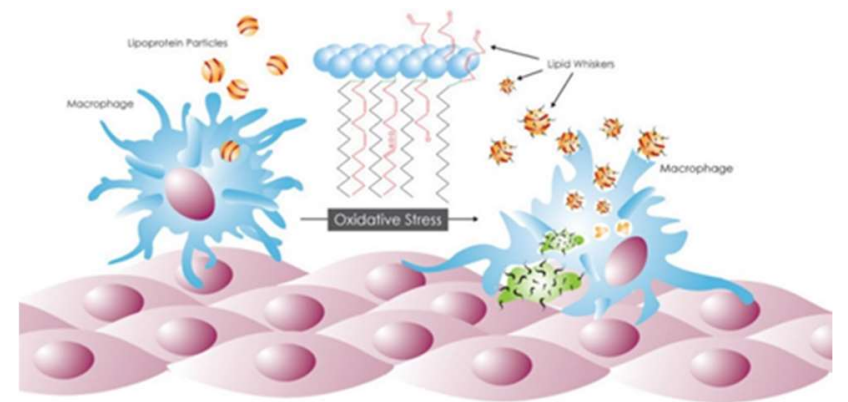

Figure 12. Oxidized Phospholipids.
(Figure13)

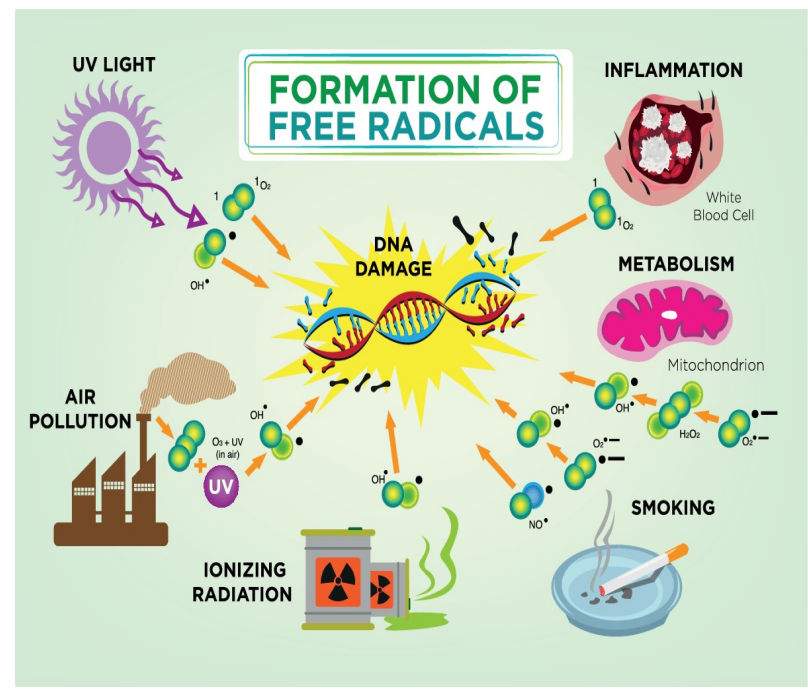

Figure 13. Formation of free radicals.

DNA damage, largely owing to oxidative stress, is a leading cause of defective sperm function. [7] (High levels of oxidative stress result in damage to sperm DNA, RNA transcripts, and telomeres and, therefore, might provide a common underlying aetiology of male infertility and recurrent pregnancy loss, in addition to congenital malformations, complex neuropsychiatric disorders, and childhood cancers in children fathered by men with defective sperm cells [8] (Figure 14).

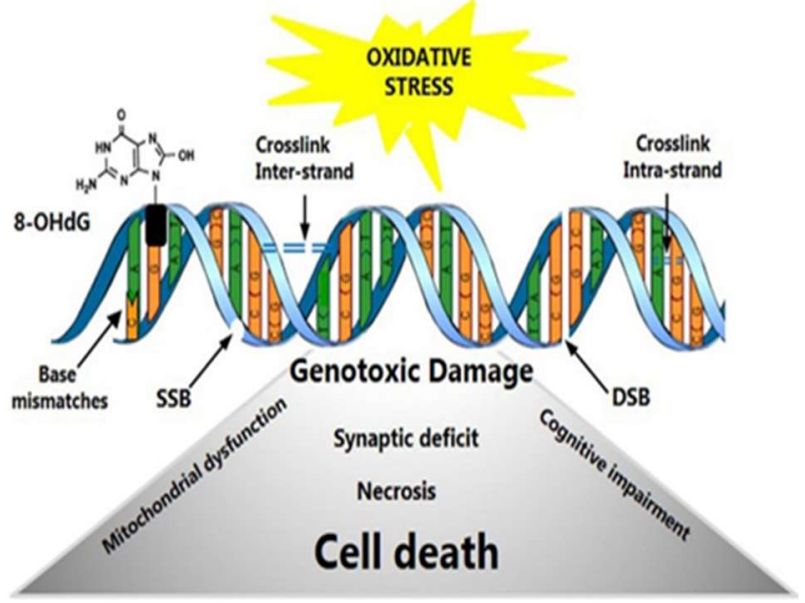

Figure 14. The effect of oxidative stress on DNA. 
The study of the mechanism of oxidative stress (Figure 15) and the regulation of oxygendependent processes has an important task for both basic and applied science.

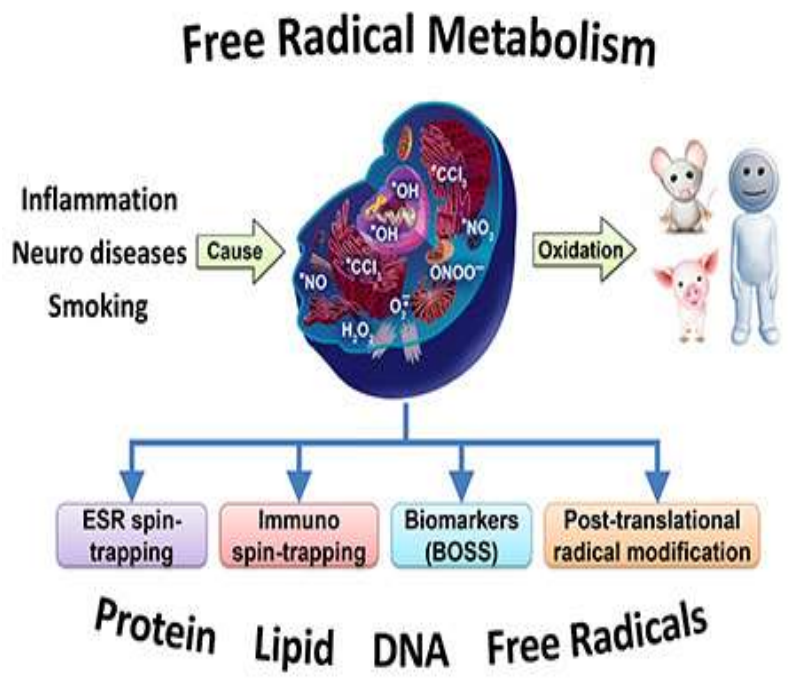

Figure 15. Mechanisms of Oxidative Stress.

Elucidation of the formation of free radical forms of oxygen (Figure 16), peroxide modification of lipids and proteins, problems of oxidative damage to biological membranes is especially important for studying their functioning [9].

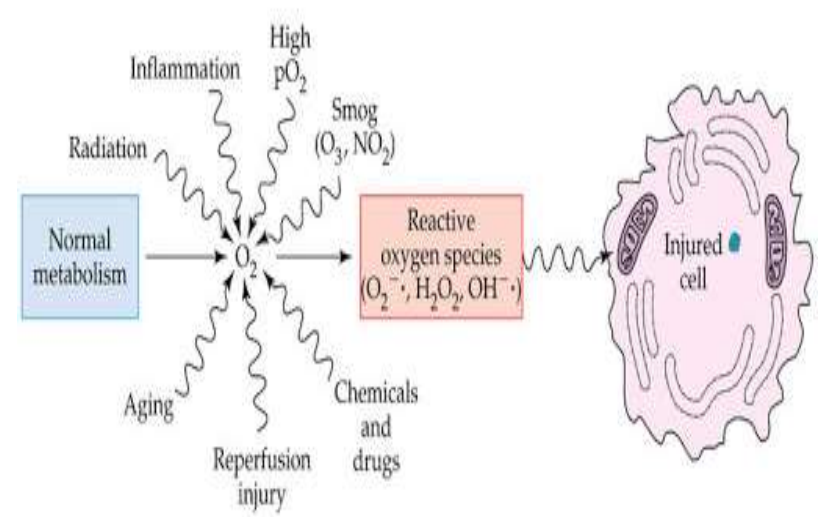

Figure 16. Formation of reactive oxygen species.

Autophagy is a catabolic process aimed at recycling cellular components and damaged organelles in response to diverse conditions of stress, such as nutrient deprivation, viral infection and genotoxic stress (Figure 17). [10]

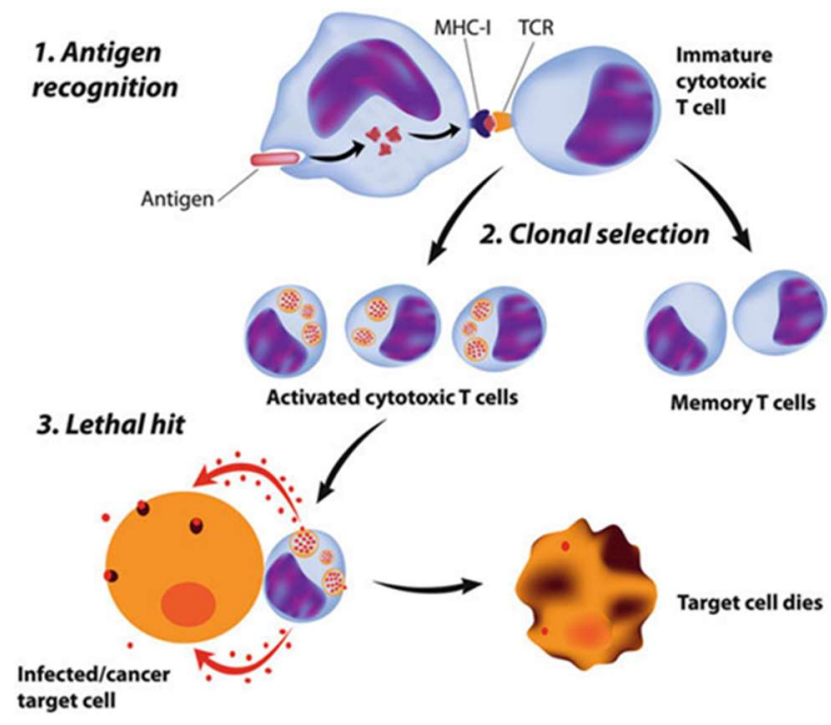

Figure 17. Cytotoxic T cell activation and action.

A growing amount of evidence in recent years argues for oxidative stress acting as the converging point of these stimuli, with reactive oxygen species (ROS) and reactive nitrogen species (RNS) being among the main intracellular signal transducers sustaining autophagy [11].

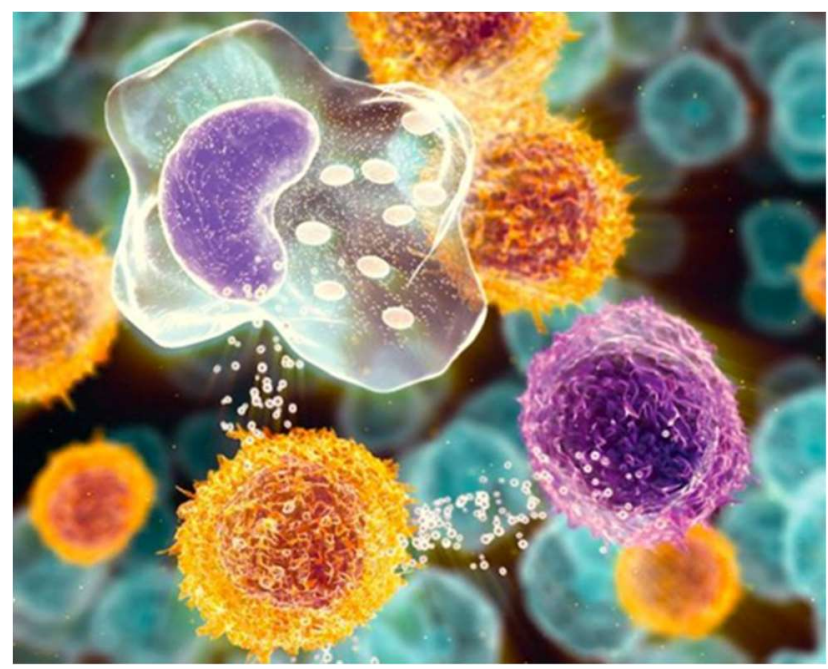

Figure 18. Reactive oxygen species.

Lipid peroxidation can occur as a consequence of tissue injury in many disease states and may 
sometimes contribute significantly to worsening the tissue injury [12]. Many assays are available to measure lipid peroxidation, but no single assay is an accurate measure of the whole process [13]. Biomarkers are needed for assessment of oxidative stress status in vivo and also for health examination, diagnosis at early stage, prognosis, safe and efficient drug development, and evaluation of efficacy of drugs, foods, beverages, and supplements. [14].

Establishing a complex interaction between oxidative stress, oxidative protein damage and the body's antioxidant defense system makes it possible to clarify the metabolic pathways of disease pathogenesis, the action of pharmacological drugs and on this basis to offer adequate, scientifically sound antioxidant therapy.

\section{Experimental part}

95 practically healthy people were examined - 50 men $(\mathrm{H})$ and 45 women $(\mathrm{F})$ of the control group (K) and 162 patients with COPD aged 18-50 years. Bacterioscopic and cytological studies were performed in preparations of scrapings of the mucous membrane of the genitals, stained by the method of RomanovskyGims. Biochemical research methods were performed in an accredited clinical diagnostic laboratory ZMAPO.

The intensity of LPO was assessed by the concentration of MDA by reaction with thiobarbituric acid.
Adhesion, ADP aggregation and disaggregation of $\operatorname{Tr}$ peripheral blood in rich $\mathrm{Tr}$ plasma were studied to determine the state of $\mathrm{Tr}$ hemostasis. In patients with COPD CO was determined by the content of the vascular component of PV contained in poor Tr plasma using formalized $\mathrm{Tr}$ donors by the method of Evans et Osten in the modification of OA Brick.

All subjects underwent a general clinical blood test: counted the number of erythrocytes, leukocytes, Tr in the counting chamber Goryaev, determined the concentration of hemoglobin in the blood, color index, calculated the leukogram by a unified method, determined ESR. Conducted morphological examination of blood plasma cells in smears stained by the method of Romanovsky-Gims. To assess the local systemic reactivity of the organism used a set of cytochemical parameters of leukocytes. The activity of myeloperoxidase (MP) PMYAL, which characterizes the oxygen-dependent mechanisms of phagocytosis, was studied by the Graham-Knollet method, and the average cytochemical coefficient (SCC) was calculated. To assess the activity of oxygen-independent mechanisms of PMYL killing, the content of cationic proteins (CB) was studied by the method of V.G. Shubich, calculated SCC. To assess the functions of Mo, the activity of the enzyme bnaphthyl esterase (NE) was determined by the Leffler method. HPLC of esterapositive lymphocytes (Li) (probably T-Li) in the total Lee pool was also calculated. Based on the data of 
leukograms and ESR of peripheral blood, samples with pairwise related variants. integrated parameters were calculated using Correlation analysis was used to numerically mathematical formulas.

The study examined 95 practically healthy individuals - K 50 men $(\mathrm{H})$ and 45 women $(\mathrm{F})$ aged 18-50 years, which were divided into groups: K1 (18-28 years) - $15 \mathrm{H}$ and $15 \mathrm{~F}$; K2 (29-39 years) - $20 \mathrm{H}$ and $15 \mathrm{~F}$; K3 (40-50 years) - $15 \mathrm{H}$ and $15 \mathrm{~F}$; 162 patients with CKD CO, in whom the duration of the disease was 2-20 years and previous treatment was ineffective. Patients were also divided into age and sex groups. Of the 162 patients with COPD CO $74-\mathrm{H}$ and $88-\mathrm{F}$, were distributed: $18-28$ years -60 patients $(22 \mathrm{H}$ and $38 \mathrm{~F}$ ), 29-39 years - 58 patients ( $30 \mathrm{H}$ and 28 F), 40-50 years - 44 patients (22 $\mathrm{H}$ and $22 \mathrm{~F})$. According to the anamnesis of life, disease and clinical examination, patients were divided into homogeneous groups. The examined patients (180 people) were also divided into homogeneous groups depending on the presence of different types of pathogens in the CO: 1 st group - patients with bacterial flora, 2nd group patients with Trichomonas, 3rd group - with fungal flora, 4th group - with chlamydial, 5th group - with viral and 6th group with mixed flora. The mean age of patients in the groups was 28.5 - 35.3 years.

We performed statistical processing of variation series, determined the criteria of reliability of differences between the two phenomena, using the statistical criterion " $\mathrm{t}$ " Student for two unrelated samples and for characterize the relationship between different parameters. Calculations were performed using Excel and Statistics software packages.

\section{Results and discussion}

In the study of microscopic examinations of swabs from the mucous membrane of the genitals of patients with chronic nonspecific inflammatory diseases of the genitals of different age groups, an inflammatory reaction was observed, the type and severity of which depended weakly on the degree of pathogenic contamination and epithelial dystrophy. The number of leukocytes ranged from 5 to 20 in the field of view, detritus and mucus were determined. No significant differences in the ratio of different types of leukocytes in groups of patients were found (Table 1). 
Table 1. The content of leukocytes in smears of scrapings from the mucous membrane of the genitals in patients with chronic nonspecific inflammatory diseases of the genitals.

Notes: $1 \mathrm{~h}$ - men of the 1 st group,

$1 \mathrm{~g}-$ women of the 1 st group,

\begin{tabular}{|l|l|l|l|l|l|l|}
\hline \multirow{2}{*}{ Indicator } & \multicolumn{5}{|c|}{ Group } \\
\cline { 2 - 7 } & $1 \mathrm{~h}$ & $2 \mathrm{~h}$ & $3 \mathrm{~h}$ & $1 \mathrm{~g}$ & $2 \mathrm{~g}$ & $3 \mathrm{~g}$ \\
\hline The & 91. & 91. & 88. & 88. & 85. & 88. \\
number of & $1 \pm$ & $9 \pm$ & $2 \pm$ & $5 \pm$ & $1 \pm$ & $6 \pm$ \\
neutrophil & 3.1 & 3.5 & 6.7 & 5.8 & 7.2 & 4.3 \\
s,\% & & & & & & \\
\hline The & 6.4 & 6.0 & 8.9 & 8.1 & 11. & 7.4 \\
number of & $5 \pm$ & $0 \pm$ & $2 \pm$ & $1 \pm$ & 66 & $1 \pm$ \\
lymphocyt & 3.1 & 2.9 & 5.3 & 4.4 & \pm & 3.3 \\
es,\% & 7 & 3 & 8 & 0 & 6.5 & 0 \\
\hline The & 2.1 & 2.1 & 2.6 & 3.2 & 2.9 & 4.1 \\
number of & $4 \pm$ & $0 \pm$ & $7 \pm$ & $4 \pm$ & $3 \pm$ & $4 \pm$ \\
histiocytes & 1.4 & 1.3 & 1.8 & 2.2 & 1.9 & 2.7 \\
,$\%$ & 6 & 3 & 8 & 2 & 9 & 1 \\
\hline
\end{tabular}

$2 \mathrm{~h}$ - men of the $2 \mathrm{nd}$ group,

$2 \mathrm{~g}$ - women of the 2 nd group,

$3 \mathrm{~h}$ - men of the $3 \mathrm{rd}$ group,

$3 \mathrm{~g}$ - women of the 3rd group.

When dividing patients into groups depending on the type of sexually transmitted infection in group 2 (with trichomoniasis) compared to others, there were probably lower rates of the relative number of lymphocytes and monocytes in smears from the genital mucosa. That is, local protective reactions, even in the chronic course of inflammation in trichomoniasis to a greater extent occur due to microphagocytosis.
Cytochemical parameters of circulating leukocytes in clinically healthy women and men were at the same level.

In patients with chronic non-specific inflammatory diseases of the genital organs, the average cytochemical coefficient of myeloperoxidase in the circulating pool of polymorphonuclear leukocytes was significantly reduced compared with $K$, except for the 1 st group of women. Thus, in patients of men of the 1st, 2nd and 3rd groups the average cytochemical coefficient of myeloperoxidase of polymorphonuclear leukocytes was reduced in $1,16,1,16$ and 1,10times, respectively $(p<0,05)$. In women of the 2 nd and 3 rd groups it was reduced to 1.10 and 1.17 times, respectively ( $p$ $<0,05$ ) (Figure 19).

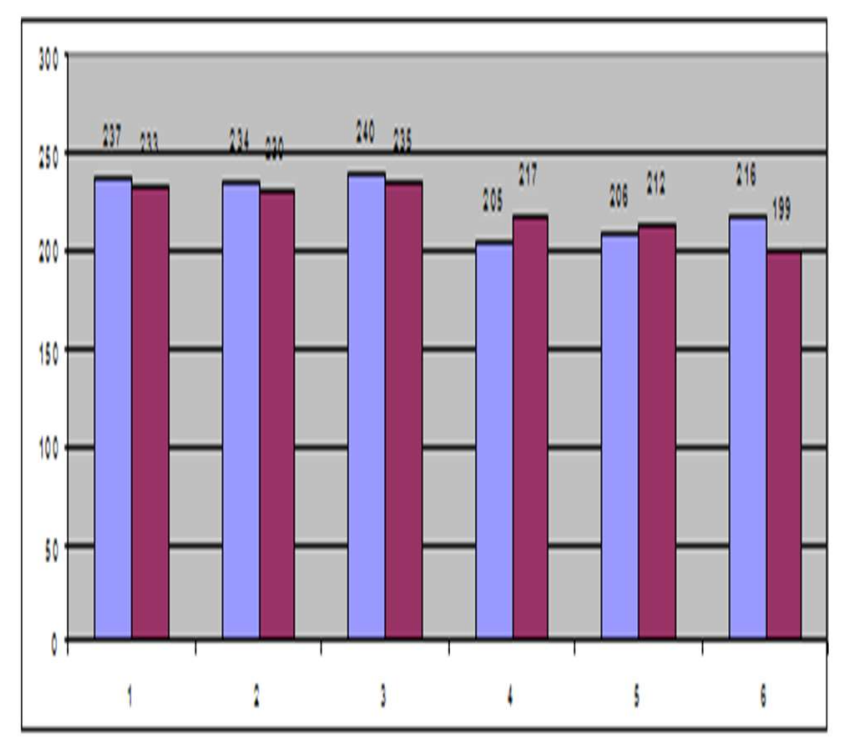

Figure 19. The average cytochemical coefficient of myeloperoxidase activity of peripheral blood neutrophils 
in patients with chronic nonspecific inflammatory diseases

of the genital organs

Notes:

\section{1-3- men K1-K3;}

2. 1'-3'.- women K1-K3;

3. 4-6- men of the 1st, 2 nd, 3rd group of patients;

4. 4'-6 '.-- women of the 1 st, 2 nd, 3 rd group of patients;

5. $\Gamma^{\mp}$ - standard deviation from the mean value.

The average cytochemical coefficient of cationic proteins of the circulating pool of polymorphonuclear leukocytes was significantly reduced in men of the 2 nd group by 1.18 times. The obtained data indicate a decrease in the phagocytic function of circulating polymorphonuclear leukocytes by both oxygendependent and oxygen-independent mechanisms.

The percentage of positively reacting cells of circulating pool lymphocytes of naphthylacetate testerase was increased in both men and women of all groups. Thus, in patients of men of the 1st, 2nd and 3rd groups, the percentage of positively responding cells of naphthylacetate testerase lymphocytes was increased by $1.28,1.25$ and 1.34 times, respectively ( $p<0,05)$ (Figure 20). In women of the 1st, 2nd and 3rd groups, it exceeded this K by $1.27,1.27$, and 1.28 times, respectively $(\mathrm{p}<0.05)$. This indicates an increase in the relative content of probably T-lymphocytes in the circulating pool of lymphocytes.

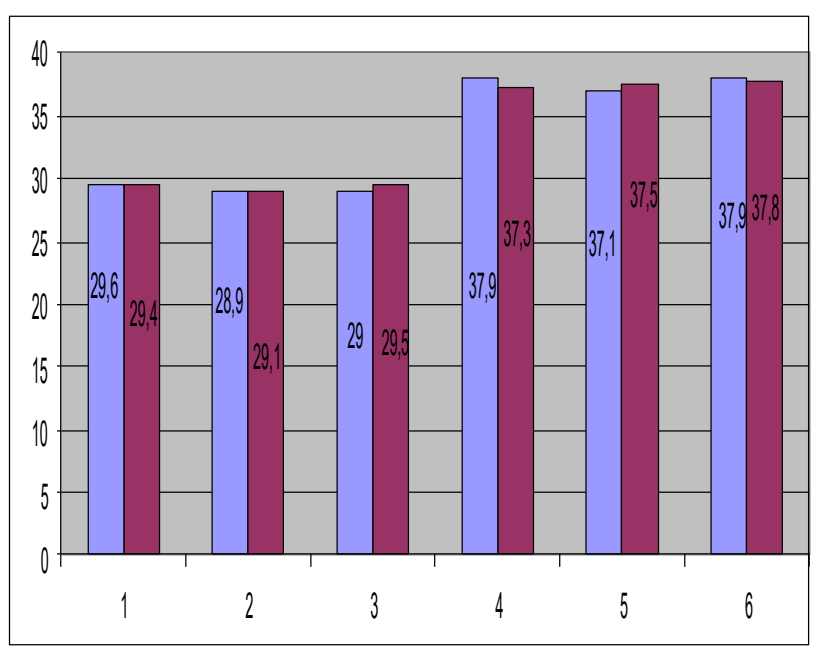

Figure 20. Percentage of positive cells for nonspecific esterases in a pool of circulating peripheral blood lymphocytes in patients with chronic nonspecific inflammatory diseases of the genital organs

Regarding the percentage of positively reacting cells of the circulating pool of monocytes in naphthylacetate testerase, a significant increase was observed in men of the 1st and 2nd groups and in women of the 3rd group in 1.22, 1.18 and 1.21 times, respectively $(\mathrm{p}<0,05)$.

In groups of patients with various types of sexually transmitted infections is determined by the preservation of myeloperoxidase activity.

In groups of patients with various types of sexually transmitted infections, a decrease in the content of cationic proteins in the circulating pool of polymorphonuclear leukocytes in groups $4,5,6(1.13 ; 1.11$ and 1.12 times $)$ is determined.

Regarding the activity of non-specific esterase of circulating monocytes, it was 
significantly increased in patients of all groups with various sexually transmitted infections, 1.2 times compared with $\mathrm{K}$.

Thus, in patients with chronic nonspecific inflammatory diseases of the genital organs with various types of sexually transmitted infections, there was a decrease in phagocytic activity of neutrophils by oxygen-independent type in groups of patients with chlamydial, viral and mixed flora. Phagocytic activity of circulating monocytes and the relative content of probable $\mathrm{T}$ - lymphocytes in the circulating pool of lymphocytes was likely to increase in patients of all groups with various sexually transmitted infections.

The average cytochemical coefficient of myeloperoxidase of tissue polymorphonuclear leukocytes in sick men did not differ significantly from that in K. (Figure 21).

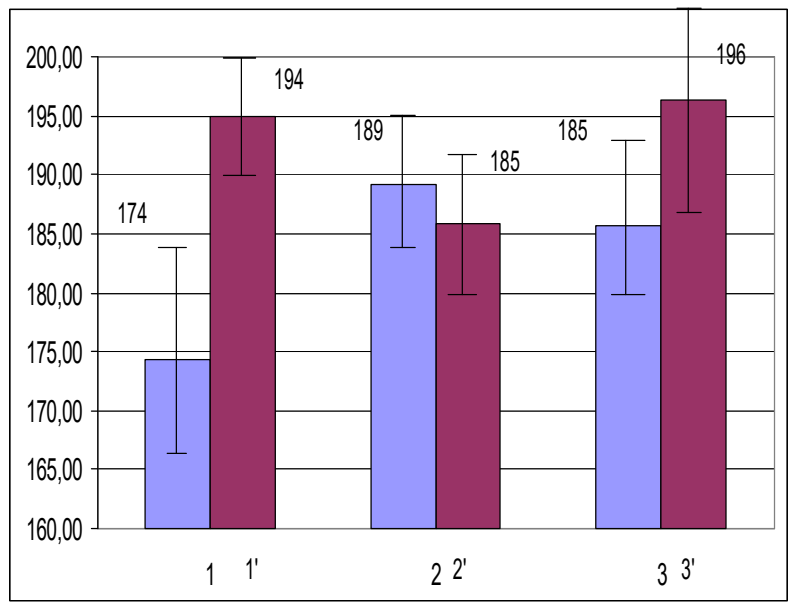

Figure 21. The average cytochemical coefficient of myeloperoxidase activity of tissue neutrophils in patients with chronic nonspecific inflammatory diseases of the genital organs.

Notes:

1. 1-3. men of 1-3 groups;
1. 1'-3'. - women of 1-3 groups;

3. $\mp^{-}$- standard deviation from the mean value.

The average cytochemical coefficient of cationic proteins of the tissue pool of polymorphonuclear leukocytes in all groups of patients with chronic nonspecific inflammatory diseases of the genital organs was significantly ( $p$ $<0.05)$ lower, on average 1.2 times lower than circulating in (Figure 22).

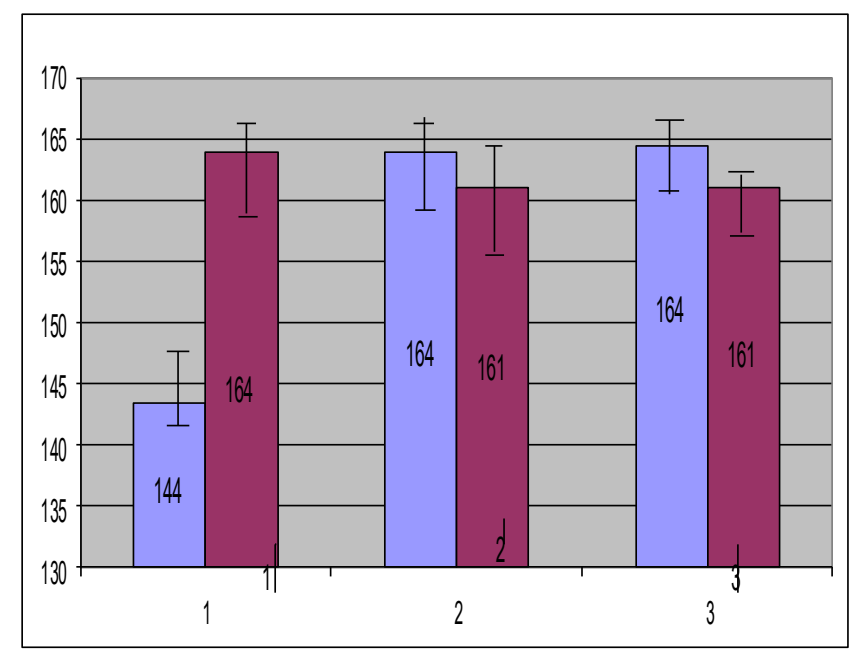

Figure 22. Average cytochemical ratio of cationic proteins in tissue neutrophils in patients with chronic nonspecific inflammatory diseases of the genital organs.

This indicates a decrease in the phagocytic activity of tissue polymorphonuclear leukocytes by an oxygen-independent mechanism compared with the activity of circulating polymorphonuclear leukocytes.

At the same time, the percentage of positively reacting cells of naphthylacetate testerase of tissue lymphocytes in all groups of patients was significantly on average 1.6 times ( $\mathrm{p}$ $<0.05)$ higher than that in the circulating pool (Figure 23). 


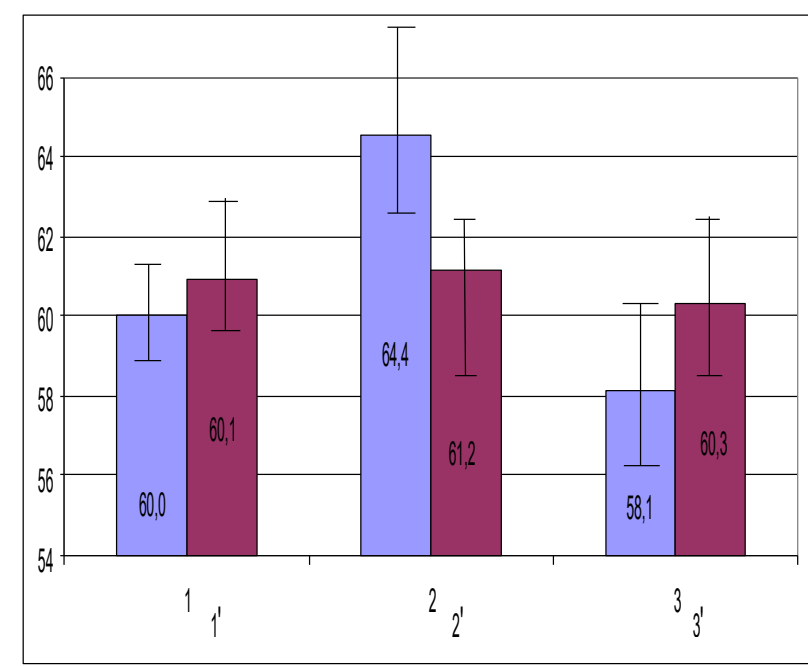

Figure 23. Percentage of positively responding cells to non-specific esterases in a pool of peripheral blood tissue lymphocytes in patients with chronic nonspecific inflammatory diseases of the genital organs.

That is, in the inflammatory center the relative maintenance of probably T-lymphocytes in a pool of lymphocytes in comparison with circulating blood is increased.

In patients with various types of sexually transmitted infections, there was a decrease in the average cytochemical coefficient of myeloperoxidase of tissue polymorphonuclear leukocytes, which was probably lower than that compared with the circulating pool of polymorphonuclear leukocytes in the same group of patients. In the 4th group of patients, the mean cytochemical coefficient of myeloperoxidase of polymorphonuclear leukocytes was probably lower than that in patients of groups 1-3 and 5-6 $(\mathrm{p}<0.05)$ (Figure 24).

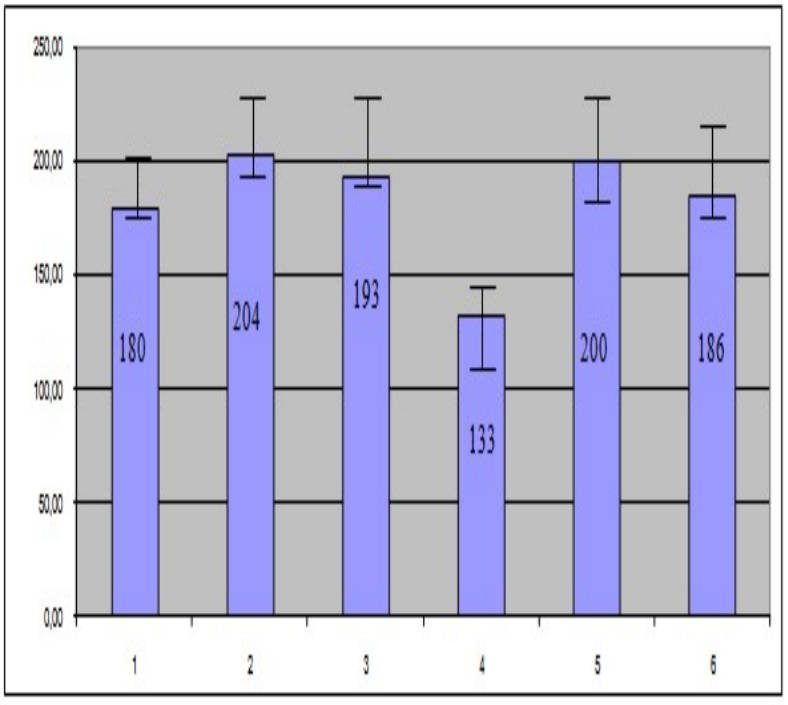

Figure 24. The average cytochemical coefficient of myeloperoxidase activity of tissue neutrophils in patients with chronic nonspecific inflammatory diseases of the genital organs with different types of sexually transmitted infections

The average cytochemical coefficient of cationic proteins of the tissue pool of polymorphonuclear leukocytes in all groups of patients with various sexually transmitted infections was also lower than that in the circulating pool by an average of 1.2 times ( $p$ $<0.05$ ) (Figure 25).

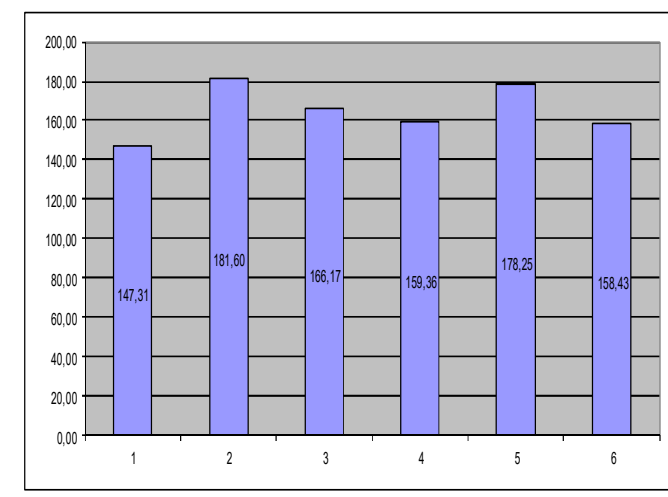

Figure 25. The average cytochemical coefficient of cationic proteins of tissue neutrophils in patients with chronic nonspecific inflammatory diseases of the genital organs with different types of sexually transmitted infections. 
However, in patients of the second group (with trichomoniasis) this indicator remained significantly higher than the same indicator in the first, third, fourth and sixth groups $(\mathrm{p}<0.05)$.

The percentage of positively responding cells to nonspecific esterases in the tissue pool of lymphocytes was equal to that in the circulating pool $(\mathrm{p}<0,05)$ (Figure 26).

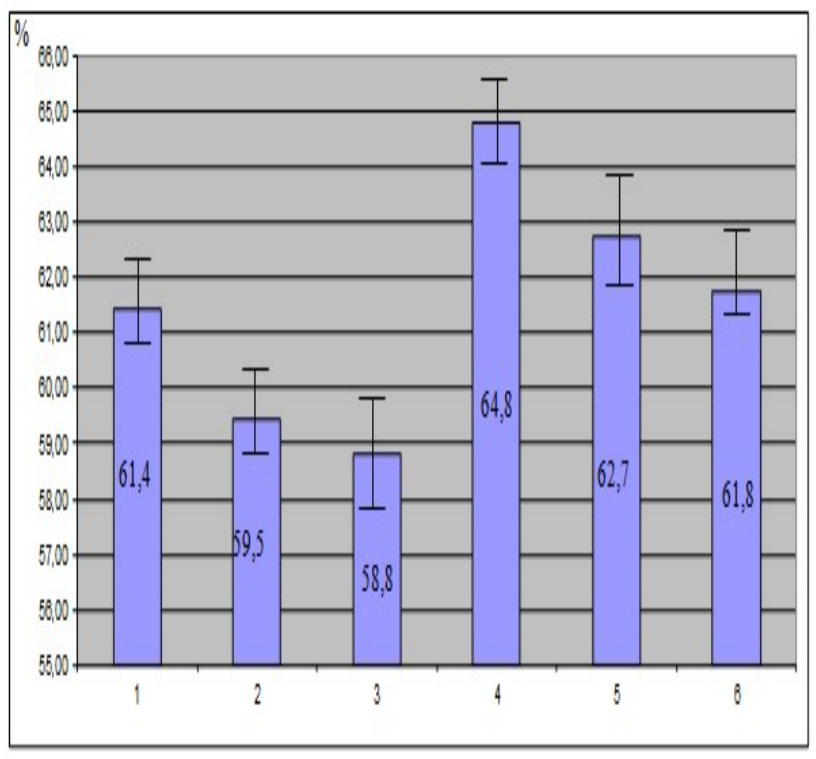

Figure 26. The percentage of positively responding cells to nonspecific esterases in the tissue poolcurrent lymphocytes in patients with chronic nonspecific inflammatory diseases of the genital organs with various types of sexually transmitted infections.

\section{Conclusions}

A comparative study of the activity of markers of oxidative stress in chronic nonspecific inflammatory diseases of the genital organs was performed. It is established that the development of chronic nonspecific inflammatory diseases of the genital organs is accompanied by the activation of oxidation of lipids and proteins. The activity of these

processes is due to insufficient antioxidant protection by reducing the content of antioxidants in prolonged oxidative stress and the activation of immunological reactions involving pro-inflammatory cytokines. This should be taken into account when correcting oxidative stress in patients with chronic nonspecific inflammatory diseases of the genital organs.

Thus, in our study, we concluded that patients with chronic nonspecific inflammatory diseases of the genitals of men and women identified a weak inflammatory reaction both systemically and locally in the lesion.

$$
\text { Phagocytic activity }
$$

of polymorphonuclear leukocytes is reduced mostly by the oxygen-dependent mechanism, and tissue - by the oxygen-independent. As an exception in patients with chlamydial infection, the phagocytic activity of tissue polymorphonuclear leukocytes was also reduced by an oxygendependent mechanism. In patients with trichomoniasis, the oxygen-independent mechanisms of phagocytosis were maintained at the highest level. In patients with chronic nonspecific inflammatory diseases of the genital organs, the predominance of the macrophage component of the phagocytosis system was found, among the cells of the immune system probably dominated by $\mathrm{T}$ - lymphocytes.

\section{Acknowledgments}


Please acknowledge anyone who contributed towards the article by making substantial contributions to conception, design, acquisition of data, or analysis and interpretation of data, or who was involved in drafting the manuscript or revising it critically for important intellectual content, but who does not meet the criteria for authorship.

\section{References}

[1] Aditya Dandekar, Roberto Mendez, Kezhong Zhang. Cross Talk Between ER Stress, Oxidative Stress, and Inflammation in Health and Disease. Methods Mol Biol. 2015; 1292: 205-14.

[2] Nakshi Sinha, Pradeep Kumar Dabla. Oxidative Stress and Antioxidants in Hypertension-A Current Review. Curr Hypertens Rev. 2015; 11 (2): 132-42.

[3] Ajoe John Kattoor, Naga Venkata K. Pothineni, Deepak Palagiri, Jawahar L. Mehta. Oxidative Stress in Atherosclerosis. Curr Atheroscler Rep. 2017 Sep 18; 19 (11): 42.

[4] Aurelia Magdalena Pisoschi, Aneta Pop. The Role of Antioxidants in the Chemistry of Oxidative Stress: A Review. Eur J Med Chem. 2015 Jun; 5 (97): 5574.

[5] Shilpa Bisht, Muneeb Faiq, Madhuri Tolahunase, Rima Dada. Oxidative Stress and Male Infertility. Nat Rev Urol. 2017 Aug; 14 (8): 470-485.

[6] Helmut Sies. Oxidative Stress: A Concept in Redox Biology and Medicine. Redox Biol. 2015; 4: 180-3.

[7] G. Filomeni, D. De Zio, F. Cecconi. Oxidative Stress and Autophagy: The Clash Between Damage and Metabolic Needs. Cell Death Differ. 2015 Mar; 22 (3): $377-88$.

[8] Ajoe John Kattoor, Naga Venkata K. Pothineni, Deepak Palagiri, Jawahar L. Mehta. Oxidative Stress in Atherosclerosis. Curr Atheroscler Rep. 2017 Sep 18; 19 (11): 42.
[9] Nakshi Sinha, Pradeep Kumar Dabla. Oxidative Stress and Antioxidants in Hypertension-A Current Review. Curr Hypertens Rev. 2015; 11 (2): 132-42.

[10] Michael M. Gaschler, Brent R. Stockwell. Lipid Peroxidation in Cell Death. Biochem Biophys Res Commun. 2017 Jan 15; 482 (3): 419-425.

[11] F. Gueraud, M. Atalay, N. Bresgen, A. Cipak, PM Eckl, L. Huc, I. Jouanin, W. Siems, K. Uchida. Chemistry and Biochemistry of Lipid Peroxidation Products. Free Radic Res. 2010 Oct; 44 (10): 1098-124.

[12] Graham J. Burton, Eric Jauniaux. Oxidative Stress. Best Pract Res Clin Obstet Gynaecol. 2011 Jun; 25 (3): 287-99.

[13] Kagan VE Lipid peroxidation in biomambrans.- Boca Raton, Florida: CRC Press; Inc., 1988. - 181p.

[14] G. Filomeni, D. De Zio, F. Cecconi. Oxidative Stress and Autophagy: The Clash Between Damage and Metabolic Needs. Cell Death Differ. 2015 Mar; 22 (3): $377-88$

[15] .Neubauer RA Free radicals and antioxidants // Lancet. - 1994. - Vol.19, №344 - P.1440-1441.Ackermann L.

[16] Brooks M. Hybertson, Bifeng Gao, Swapan K. Bose, Joe M. McCord. Oxidative Stress in Health and Disease: The Therapeutic Potential of Nrf2 Activation. Mol Aspects Med. 2011 Aug; 32 (4-6): 234-46.

[17] Aditya Dandekar, Roberto Mendez, Kezhong Zhang. Cross Talk Between ER Stress, Oxidative Stress, and Inflammation in Health and Disease. Methods Mol Biol. 2015; 1292: 205-14.

[18] C. Wright, S. Milne, H. Leeson. Sperm DNA Damage Caused by Oxidative Stress: Modifiable Clinical, Lifestyle and Nutritional Factors in Male Infertility. Reprod Biomed Online. 2014 Jun; 28 (6): 684-703.

[19] AM Hruszkewycz. Evidence for Mitochondrial DNA Damage by Lipid Peroxidation. Biochem Biophys Res Commun. 1988 May 31; 153 (1): 191-7.

[20] JM Gutteridge, B. Halliwell. The Measurement and Mechanism of Lipid Peroxidation in Biological Systems. Trends Biochem Sci. 1990 Apr; 15 (4): 129-35. 
[21] Moore, K.J.,Tabas, I. Macrophages in the pathogenesis of atherosclerosis. Cell 145; 2011, pp. 341355.

[22] Vizir VA, Makurina GI. State of free-radical processes and antioxidant defence of patients with psoriasis and concomitant essential hypertension. Zaporozhye Medical Journal. 2016(4):21-8. 